\title{
Herniorrafía con la técnica de Lichtenstein versus Nyhus en el manejo de las hernias inguinales y sus complicaciones postoperatorias - Réplica
}

\section{Hernia repair comparing Lichtenstein and Nyhus techniques for the management of inguinal hernia and its postoperative complications - Reply}

Correspondencia Antonio Quispe drantonioquispe@gmail.com

Recibido: 08/12/2016 Aprobado: 14/12/2016

Citar como: Granda AC, Correa-Tineo S, Quispe AM. Herniorrafía con la técnica de Lichtenstein versus Nyhus en el manejo de las hernias inguinales y sus complicaciones postoperatorias - Réplica. Acta Med Peru. 2016;33(4):340-1

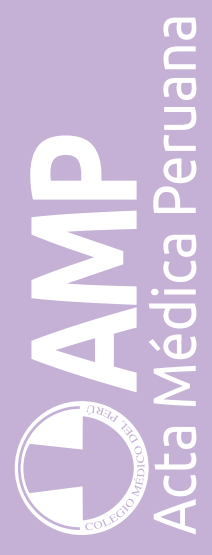

\author{
Ana C. Granda', Santos Correa-Tineo ${ }^{1,2}$, Antonio M. Quispe 3,4 \\ 1 Facultad de Medicina, Universidad Nacional Mayor de San Marcos. Lima, Perú. \\ 2 Servicio de Cirugía, Hospital Militar Central Luis Arias Schreiber. Lima, Perú. \\ 3 Department of International Health, Johns Hopkins Bloomberg School of Public Health. Baltimore, EE.UU. \\ 4 Grupo de Investigación Biomédica, Web Med Research. Lima, Perú.
}

Nos gustaría agradecer el interés en nuestro artículo titulado "Herniorrafía con la técnica de Lichtenstein versus Nyhus en el manejo de las hernias inguinales y sus complicaciones postoperatorias en un hospital del Perú" [1]. Nosotros estamos muy gratificados de poder continuar discutiendo y respondiendo a sus comentarios.

Con respecto al comentario de Robles-Valcárcel et al., este nos permite aclarar algunos aspectos metodológicos de nuestro estudio $y$, en general de los estudios trasversales, que bien vale la pena explicar. Primero, la naturaleza incidente o prevalente de una variable outcome o desenlace de interés no depende del diseño de estudio y viceversa. Esto se debe a que un desenlace es incidente en la medida que los investigadores al medirlo pueden discernir si está frente a un evento nuevo (incidente) o no (prevalente) ${ }^{[2]}$. Segundo, la naturaleza incidente de las complicaciones posquirúrgicas como outcome o variable desenlace de interés es indubitable. Podemos decir esto sin temor a equivocarnos porque el periodo de riesgo de las complicaciones post quirúrgicas tiene un inicio claro (se inicia con la cirugía) y su ocurrencia previa a la cirugía es una imposibilidad estadística. Tercero, nuestro estudio es de diseño trasversal porque la medición de la exposición y los eventos de interés fueron medidos en un solo momento (entiéndase en un solo corte trasversal en el tiempo) ${ }^{[3]}$. Dicho esto, vale la pena explicar que para que un estudio sea de diseño tipo cohortes primero tiene que medirse la exposición (medición basal que permite clasificar a la población de estudio en expuestos y no expuestos) y luego en una o más mediciones de seguimiento cuántos expuestos y no expuestos manifestaron o no el evento o los eventos desenlace de interés ${ }^{[4]}$. Estos estudios pueden ser retrospectivos, prospectivos o mixtos dependiendo de si la ocurrencia de la exposición y los desenlaces de interés fueron medidos de forma retrospectiva (cohortes retrospectivas), de si ambos fueron medidos a futuro (cohortes prospectivas) o si la exposición fue medida retrospectivamente y los desenlaces de forma prospectiva (cohortes mixtas) ${ }^{[4]}$.

Nosotros agradecemos los comentarios de Cassana A. y Chang-Cabanillas $S$ et al., los cuales nos permiten resaltar algunos aspectos estadísticos de nuestro estudio que bien merecen ser comentados. Primero, dado que ningún estudio está exento de limitaciones lo importante no es 
no tener limitaciones sino saber reconocerlas e interpretar los hallazgos en el contexto de las mismas. De ahí que, como es altamente recomendado, en la discusión del manuscrito reconocemos las principales limitaciones de nuestro estudio y al momento de sacar conclusiones somos muy conservadores, sobre todo considerando que la limitada validez externa e interna del mismo no nos permite ser concluyentes. Segundo, como específicamente mencionamos en nuestra discusión "es muy probable que existan una serie de confusores no medidos en nuestro análisis, también es poco factible que el efecto de los mismos haya pasado desapercibido en nuestro análisis" (1] o que, "de haber sido incluidos, cambien radicalmente los resultados de nuestro análisis" [1]. Esto se debe a que una de las fortalezas de nuestro estudio es precisamente la rigurosidad del análisis, utilizando como método de control de confusores el análisis de regresión multivariante, respetando la granularidad de los datos sin categorizar las variables numéricas e interpretando conservadoramente nuestros hallazgos ${ }^{[5]}$. Tercero, coincidimos en que existen muchos métodos de regresión por los cuales pudimos haber optado, incluyendo los análisis de regresión de Poisson para la estimación del riesgo relativo (RR), pero tomando en consideración la baja incidencia de complicaciones, la popularidad y facilidad de interpretación del odds ratio (OR), así como la aproximación del OR al RR cuando la frecuencia del desenlace de interés es relativamente baja ${ }^{[6]}$, optamos por estimar el OR en vez del RR. Finalmente, coincidimos con los comentarios de Cassana A y Chang-Cabanillas S et al., en el sentido de que es muy importante que todo estudio que analice la asociación entre dos variables utilice algún método de control de confusión y por lo menos utilice un análisis de regresión multivariante ajustando los confusores medidos ${ }^{[7]}$, motivo por el cual optamos por este y no inhibimos de sacar conclusiones en base a análisis bivariados o crudos.

Es preciso mencionar que esta carta es una opinión de sus autores y no necesariamente refleja la opinión de sus instituciones. Declaramos que no tenemos conflictos de interés.

\section{REFERENCIAS BIBLIOGRÁFICAS}

1. Granda AC, Correa-Tineo S, Quispe AM. Herniorrafía con la técnica de Lichtentein versus Nyhus en el manejo de las hernias inguinales y sus complicaciones postoperatorias en un hospital del Perú. Acta Med Peru. 2016;33(3):208-16.

2. Noordzij M, Dekker FW, Zoccali C, Jager KJ. Measures of disease frequency: prevalence and incidence. Nephron Clin Pract. 2010;115(1): :17-20.

3. Levin KA. Study design III: Cross-sectional studies. Evid Based Dent. 2006;7(1):24-5.

4. Levin KA. Study design IV. Cohort studies. Evid Based Dent. 2006;7(2):51-2.

5. Barnwell-Menard JL, Li Q, Cohen AA. Effects of categorization method, regression type, and variable distribution on the inflation of Type-l error rate when categorizing a confounding variable. Stat Med. 2015;34(6):936-49.

6. Rigby AS. Statistical methods in epidemiology. III. The odds ratio as an approximation to the relative risk. Disabil Rehabil. 1999;21(4):145-51.

7. McNamee R. Regression modelling and other methods to control confounding. Occup Environ Med. 2005;62(7):500-6.

\section{Las ediciones anteriores de Acta Médica Peruana están disponibles en:}

\section{www.scielo.org.pe}

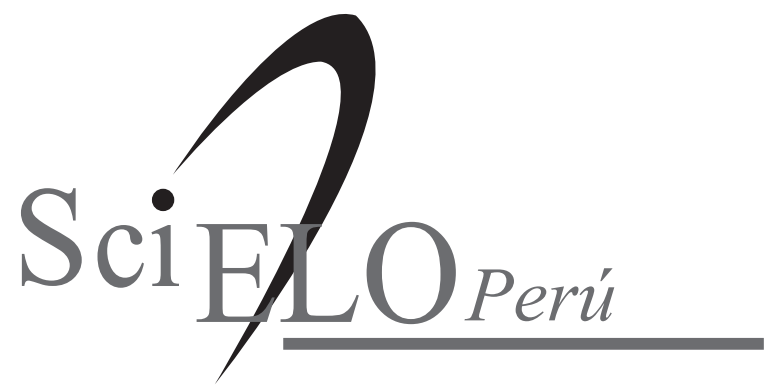

